\title{
INTERACTING BINARY WHITE DWARF STARS
}

\author{
J.-E. SOLHEIM \\ Institute of Mathematical and Physical Sciences \\ University of Tromsø \\ $N-9000$ Tromsø \\ Norway
}

\begin{abstract}
This group of stars consists of 4 systems, also called helium cataclysmics. Three of them show photometric variations and have been studied by the Whole Earth Telescope (WET), which have revealed multiperiodic light curves showing the signature of $g$-mode non-radial pulsations on the accreting star. The combination of accretion and $g$-mode pulsations gives a unique opportunity to test models for the accreator's structural changes in response to accretion. IUEspectra provide additional physical parameters.
\end{abstract}

\section{Introduction}

One of the possible final stages of close binary star evolution is a system in which a carbon-oxygen white dwarf is accreting helium from a degenerate low mass helium secondary. The accreator may end up as an ordinary DB white dwarf star when its companion is completely cannibalized.

We know today of 4 systems, which have only helium and some traces of metals in their spectra, and show sign of mass transfer by exposing a He-disk spectrum or flickering in their light curves. These systems, named Interacting Binary White Dwarf stars (Nather 1985) are AM CVn (= HZ 29), V803 Cen (= AE 1), GR Boo (= PG1346+082) and GP Com (= G21-29).

Of the 4 objects, AM CVn varies a few per cent around a mean value of $B=13.9$. V803 Cen and CR Boo varies like dwarf novae between a high and a low state, but unlike the dwarf novae, they spend most of their time in the high state. GP Com is the only object where an orbital period has been determined from spectral line modulations (Nather et al. 1981).

\section{Photometry}

From the long time-series photometry obtained with the Whole Earth Telescope project (Nather 1990) we are able to resolve fine structure in the frequency spectrum, as exemplified in Figure 1 for CR Boo (Provencal et al. 1991) and for AM CVn (Solheim et al. 1991). 
For CR Boo the strong oscillation with periods near $1490 \mathrm{~s}$ has been resolved in a band of as many as 15 separate periodicities. The dominant period of $1471.35 \mathrm{~s}$ is coherent over the WET run length (12 days), and is the first coherent periodicity detected in the system. The rich pulsation spectrum cannot be due to a simple binary model, and we may in this case observe partial ionization driven pulsations of the accreting star where part of the energy may be supplied by the accretion process.

For AM CVn we observe frequency triplets with equal frequency separation of $21 \mu \mathrm{Hz}$ (Figure 1). This can be interpreted as $g$-mode non-radial pulsations on the accreting star, where the frequency splitting is due to rotation.

The pulsation spectra observed give us for the first time, tools to investigate models for the internal structure of an accreting degenerate star.

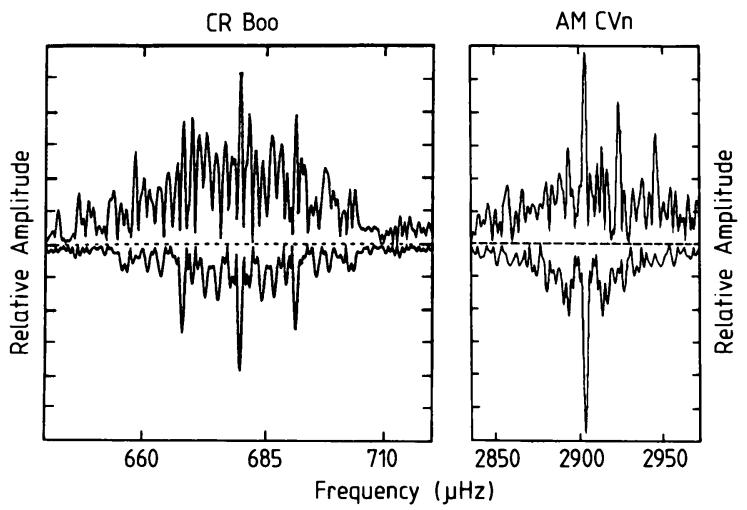

Figure 1. Amplitude-FT and window function of the low state data of CF Boo (left panel) in the region of 1490 seconds (from Provencal et al. 1991), and (right panel) the same in the region of $350 \mathrm{~s}$ for AM CVn. A window function is a single sinusoid sampled the same way as the data.

\section{IUE-Spectroscopy}

A comparison of UV-continuum and line spectra for dwarf nova in the high and low states, has shown that a majority of 80 percent of the dwarf novae during the high state have identical UV flux distribution within narrow limits, best described by the continuum spectra of B2-3 V-III stars of temperature $20000 \pm 2000 \mathrm{~K}$ (la Dous 1990).

In Figure 2 the UV flux of the IBWDs in the high and low states are compared. Except for GP Com which has a redder spectrum, the IBWDs have a bluer spectrum than the dwarf novae. This may be interpreted as due to a higher mass transfer rate, lower inclination, or the presence of a small and hot central object.

The flux distributions in the low and the high state of V803 Cen are almost identical. For CR Boo the bluest part has the same slope in the high and low states, while the high state spectrum has a stronger red component. This may imply that the outer edge of the disk is expanding and cooling when this system is in the high state. 
LOW STATE
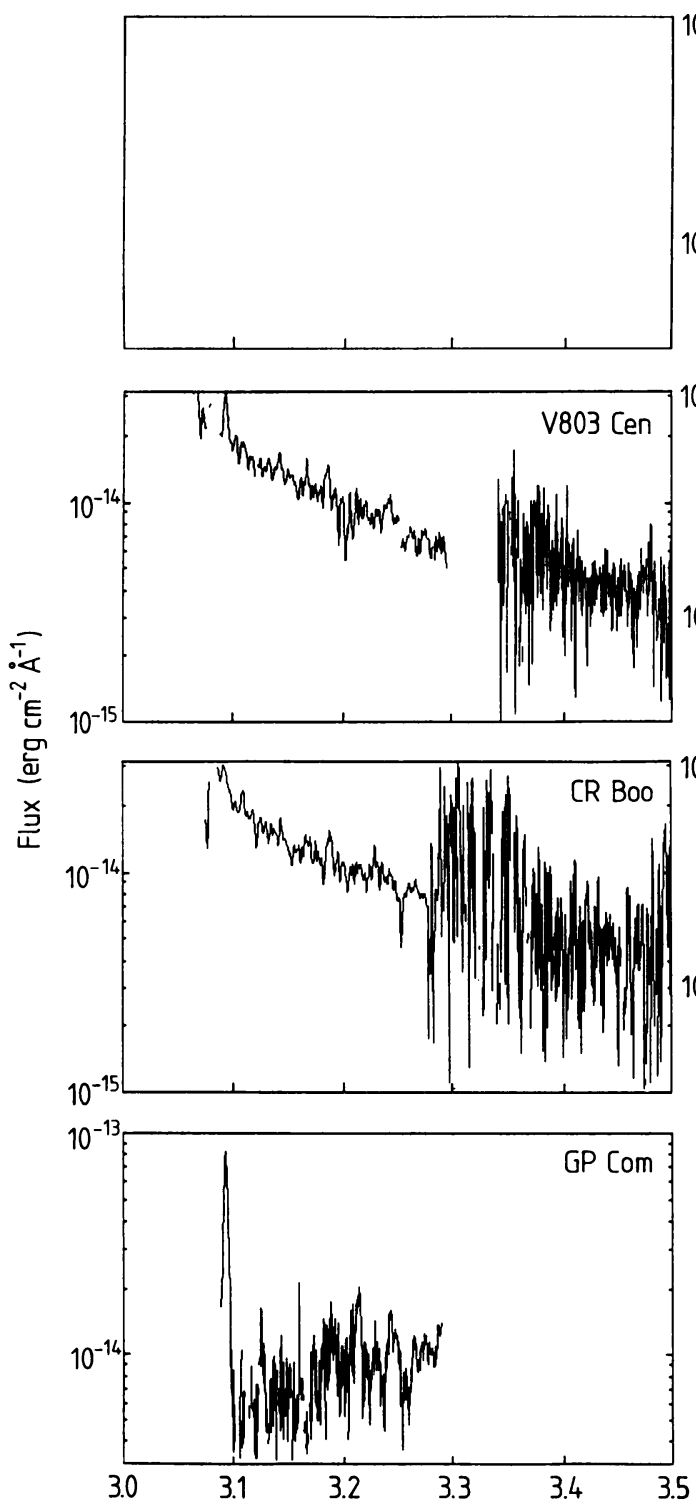

HIGH STATE
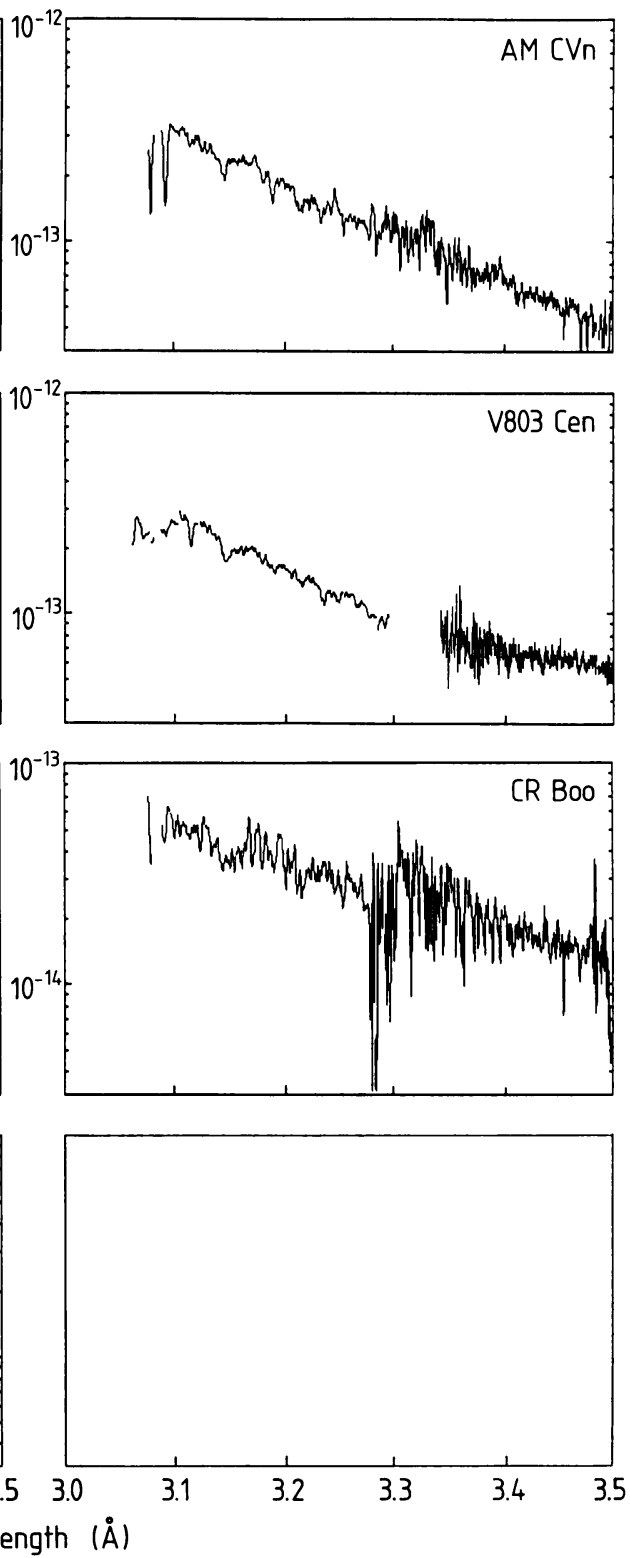

Figure 2. A comparison of representative spectra for interacting Binary White Dwarf stars in high and low states. GP Com has a quite different spectra slope than the others. (CR Boo high state spectra are taken at standstill at magnitude 15.) 
GP Com has a redder spectrum with strong emission lines. In this case mass transfer rate has been reduced, and the line-forming region is optically thin. The temperature of the hot central source is lower than for the other systems.

\section{Conclusions}

WET photometry gives us tools for investigation pulsations on the mass accreting objects in IBWD systems. UV-spectra give information about the hot central source and the expanding or contracting disk.

Further investigation of these objects may tell us whether they are progenitors of single DB white dwarfs or doomed for a more violent future as supernovae.

ACKNOWLEDGEMENTS: This research is supported by grants from the Norwegian Research Council for Sciences and Humanities. The assistance from the IUE - Vilspa staff and the use of ULDA archives is greatly appreciated.

\section{References}

la Dous, C., 1991, Astron. Astrophys., in press. (Vilspa IUE preprint no 54.)

Nather, E.L., Robinson, E.L., and Stover, R.J, 1981, Ap. J. 244, 269.

Nather, R.E., 1985, in Interacting Binaries, P.P. Eggleton and J. Pringle (eds.), NATO ANSI, series C, Vol. 150, Reidel, 349.

Nather, R.E., Winget, D.E., Clemens, J.C., Hansen, C.J., and Hine, B.P., 1990, Ap. J., 361, 309.

Provencal, J., Winget, D.E., Nather, R.E., Clemens, J.C., Hine, B.P., Henry, G., Kepler, S.O., Vauclair, G., Chevreton, M., O'Donoghue, D., Warner, B., Grauer, D.A., and Ferrario, L., 1991, in White Dwarfs, G. Vauclair and E. Sion (eds.), Kluver, 449.

Solheim, J.-E., Emanuelsen, P.-I., Vauclair, G., Dolez, N., Chevreton, M., Barstow, M., Sansom, A.E., Tweedy, R.W., Kepler, S.O., Kanaan, A., Fontaine, G., Bergeron, P., Grauer, A.D., Provencal, J.L., Winget, D.E., Nather, R.E., Bradley, C.F., Claver, C.F., Clemens, J.C., Kleinman, S.J., Hine, B.P., Marar T.M.K., Seetha, S., Ashoka, B.N., Leibowitz, E.M., and Mazeh, T., 1991, in

White Dwarfs, G. Vauclair and E. Sion (eds.), Kluver, 431. 